\title{
Saturation Transfer Ratio in Magnetic Resonance Imaging. A Novel Physical Parame- ter for Evaluation of the Hydrophilicity of Synthetic Copolymer Gels
}

\author{
Shigeru MATSUShIMA, Akinori TAKasu, Yoshihito InAI, \\ Tadamichi HIRABAYASHI, \\ Seiichi ERA, ${ }^{*}$ Masaru SOGAMI, ${ }^{*}$ and Yasutomi KINOSADA ** \\ Department of Environmental Technology and Urban Planning, \\ Graduate School of Engineering, Nagoya Institute of Technology, \\ Gokiso-cho, Showa-ku, Nagoya 466-8555, Japan \\ *Departments of Physiology and **Medical Informatics, \\ School of Medicine, Gifu University, \\ 40 Tsukasa-machi, Gifu 500-8705, Japan
}

(Received February 24, 2000; Accepted June 15, 2000)

\begin{abstract}
It was first found that there is good correlation between saturation transfer ratio (STR) values in magnetic resonance imaging (MRI) measurement and the hydrophilicity of various synthetic copolymer gels containing 2hydroxyethyl methacrylate (HEMA), glycidryl methacrylate (GMA), $N$-vinyl-2-pyrrolidinone ( $N$-VP), and/or methyl methacrylate (MMA). The hydrophilicity of these copolymer gels was characterized by water contents evaluated by differential scanning calorimeter (DSC) and also the solubility parameter in theoretical sense. The MRI measurement was performed by conventional field-echo imaging as well as under the irradiation of an off-resonance saturation pulse apart by 75,19 , and $4 \mathrm{ppm}$ from a resonance point of water. The STR values offset at the 75-ppm correlated well with the total water contents in the samples. The values offset at the 19-ppm divided the samples into two classes corresponding to hydrophilic and hydrophobic gels. In the case of 4-ppm offset, the hydrophilic gels showed significantly higher STR values than the hydrophobic ones and the values among the hydrophilic gels were reflective to the constrained water content in the gel. Moreover the values correlated linearly with the solubility parameter of the samples. Thus the STR values in measurement of MRI could be adopted as a new useful parameter for molecular or material characterization of synthetic copolymers without any invasive processes.

KEY WORDS Saturation Transfer Ratio / Magnetic Resonance Imaging / Solubility Parameter / Constrained Water / Hydrophilicity / Copolymer Gel / 2-Hydroxyethyl Methacrylate / N-Vinyl-2-Pyrrolidone / Glycidryl Methacrylate /
\end{abstract}

Nuclear magnetic resonance (NMR) technique has played an important part to elucidate many structural problems of various polymers. In conventional NMR, the resonance frequency of the special nucleus containing in a sample, for example ${ }^{1} \mathrm{H},{ }^{13} \mathrm{C}$, and so on and its relative strength are related to the structure of the sample. Some authors have investigated hydration manners in gels by using NMR. ${ }^{1-7}$ The mobility of water in the gels and their structure have been correlated by diffusion coefficient. The diffusion coefficient of water in poly(2-hydroxyethyl methacrylate) gel showed a large dependence on the degree of hydration. ${ }^{3}$ By the way, the correlation between NMR signals and conditions of water in gels has been shown by the terms of magnetization transfer (MT) as well as spin lock (SL). ${ }^{4-9}$ The MT value is due to a second ${ }^{1} \mathrm{H}$ compartment and a small fraction of rigidlattice magnetic moments in macromolecular tissue like proteins. ${ }^{12,13}$ In SL, nuclear spins are locked with a radio frequency (RF) field. ${ }^{13}$

As an alternative application of the NMR technique, magnetic resonance imaging (MRI) method has been remarkably developed in a field of clinical medicine. The MRI is distinguishable method for the tissue characterization with non-invasive modality. Most of modern hospitals adopt this novel instrument system to know a diseased part through some abnormal signs in the imaging picture. Therefore MRI must become a good method also

\footnotetext{
${ }^{\dagger}$ To whom correspondence should be addressed.
}

for characterization of synthetic copolymer gels without destroying their outward form and functions. Unfortunately this method is not so widely applied to the structural analysis of synthetic polymers. In such high resolution MRI, changing MT and SL values should selectively affect on the contrast of the picture because of different in chemical and magnetic environments of macromolecular structure. Off-resonance RF preparatory pulses have been extensively discussed in the recent literatures concerning $\mathrm{MR}$ so as to manipulate image contrast. ${ }^{14-17}$ The image contrast obtained is highly dependent on the offset frequency and is produced by three different mechanisms: spin tip $(0-3.1 \mathrm{ppm}$ from a resonance center), spin lock $(3.1-31.3 \mathrm{ppm})$, and also magnetization transfer $(0-234.4 \mathrm{ppm}) .^{10}$ The results represent significant improvements in image contrast by operating in the near-resonance frequency offset.

However, there is no report with respect to the relationship between saturation transfer ratio (STR) and hydrophilicity of any synthetic copolymer gel systems by using MRI. It is a main purpose of our present work to study the correlation between STR and traditional parameter for the hydrophilicity of synthetic copolymer gels containing the solubility parameter. Finding a satisfactory correlation must be the first step to explore a novel method by MRI for the structural/material characterization of artificial polymer gels containing water as well as polymeric tissue in vivo. 
(a)

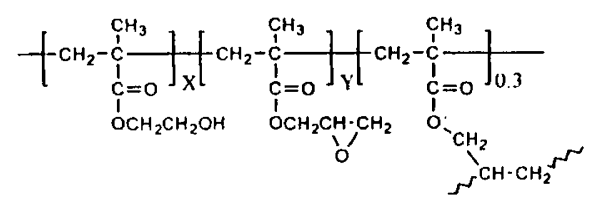

(b)

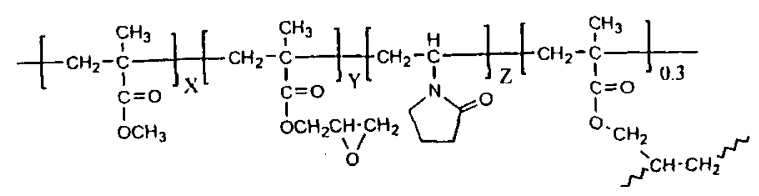

(c)

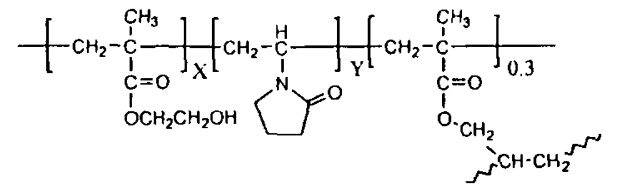

(d)

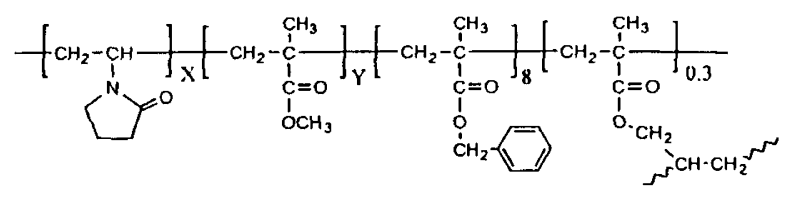

Figure 1. Molecular formula of the synthetic copolymer gels. (a) poly(HEMA-stat-GMA); (b) poly(N-VP-stat-GMA / MMA); (c) pol y(HEMA-stat-N-VP); (d) poly( N-VP-stat-MMA / BzMA).

\section{EXPERIMENTAL}

\section{Materials}

Fourteen synthetic copolymers were kindly provided by Menicon Co. Ltd., which were composed of any two or three monomers among 2-hydroxyethyl methacrylate (HEMA), grycidyl methacrylate (GMA), methyl methacrylate (MMA), benzyl methacrylate (BzMA) and $N$ vinyl-2-pyrrolidinone ( $N$-VP). These were classified into four types as illustrated in Figure 1. The chemical composition of the original copolymers could be determined by an elemental analysis. Gelation was conducted by adding a very small amount $(<0.3 \%)$ of allyl methacrylate. The sample copolymer was shaped up $15 \phi \mathrm{mm}$ columnar (length $=100 \mathrm{~mm}$ ) for MRI measurement.

\section{Determination of Water Contents}

The total water content of the synthetic copolymer gels was determined as the weight deference of the gels before and after drying-up for $18 \mathrm{~h}$ at $120^{\circ} \mathrm{C}$ and under the reduced pressure of $1-10 \mathrm{mmHg} .{ }^{18}$ Water content of the sample gels were also evaluated on the basis of data of differential scanning calorimeter (DSC), which were run on Seiko DSC 200 model. Each sample $(10 \mathrm{mg})$ was down to $-80^{\circ} \mathrm{C}$ and then heated up to $35^{\circ} \mathrm{C}$ at $5^{\circ} \mathrm{C} \min ^{-1}$ under the nitrogen atmosphere. Free and constrained water in the gels appeared as a different peak, respectively, on their DSC curves. In this study, the water condition was defined as follows. The free water was normal

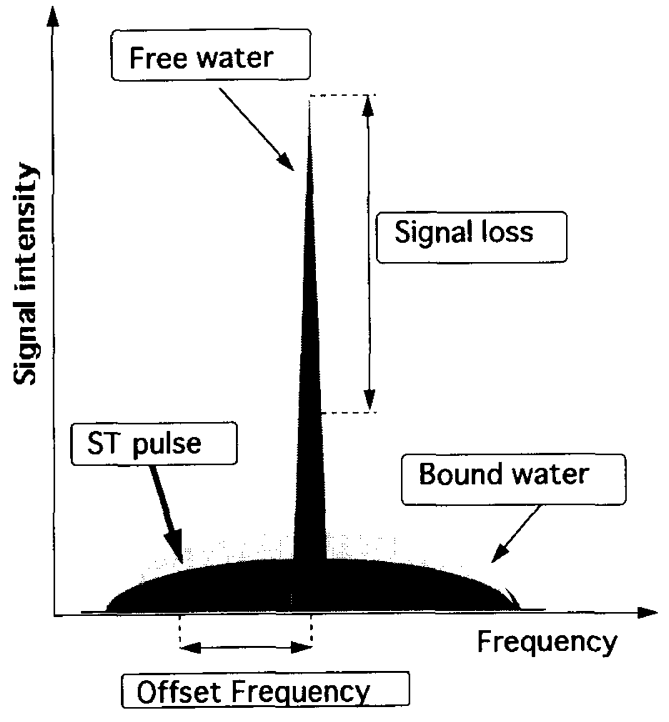

Figure 2. Scheme of saturation transfer effect. In the clinical MRI, signal intensity of free water was obtained for MR imaging. In the ST prepared image, the signal intensity of free water were deceased than without ST prepared image by ST effect. Their signal reduction was different at ST pulse frequency offsets.

freezing water without the influence of functional groups in the macromolecules and constrained water was freezing water at low temperatures than the free water owing to the influence of macromolecular matrixes. In this paper, "bound water" contains both constrained and non-freezing water.

\section{Magnetic Resonance Imaging (MRI) Measurement}

MRI picture was obtained on Signa model of GE Medical Systems equipped with 1.5 Tesla clinical scanner and birdcage-type head coil. After axial localizer images were recorded, the sample gels were scanned in the coronal plane. The coronal plane images were preformed as follows. First, we obtained $\mathrm{T}_{2}$-weighted image (repetition time $=3000 \mathrm{~ms}$, echo time $=120 \mathrm{~ms}$ ) and $\mathrm{T}_{1}$-weighted image (repetition time $=500 \mathrm{~ms}$, echo time $=11 \mathrm{~ms}$ ). Then conventional spoiled-gradient recalled acquisition in the steady state (SPGR) and also SPGR under the irradiation of ST-pulse (ST-SPGR) were obtained in the plane of the sample's center.

The off-resonance technique was adopted to achieve preferential saturation of the immobile protons and to calculate STR values. A concept of the ST-effect for offresonance technique is depicted in Figure 2. The single ST-pulse frequency in an interval of $18 \mathrm{~ms}$ was irradiated at the frequency of 75,19 , or $4 \mathrm{ppm}$ apart from that of water resonance. The off-resonance saturation pulse had a bandwidth of $110 \mathrm{~Hz}$ and flip angle of 900 degrees. The pulse amplitude was $3.6 \times 10^{-6} \mathrm{~T}$ for the STsequence. These measurement conditions have demonstrated a peak specific absorption rate (SAR) of 2.89 $\mathrm{W} \mathrm{kg}^{-1}$, which is satisfied within the FDA recommendation value of $8 \mathrm{~W} \mathrm{~kg}^{-1}$ for any tissues of $1 \mathrm{~g}$. MR imaging parameters were as follows: repetition time $=50 \mathrm{~ms}$; echo time $=5 \mathrm{~ms}$; flip angle $=30$ degree; matrix size $=$ $256 \times 192$ dots; field of view $=16 \mathrm{~cm}$; section thickness $=$ $5 \mathrm{~mm}$; intersection gap $=2 \mathrm{~mm}$; and slice number $=1$.

The regions of interest (ROI) for signal intensity were measured at the same site inside of the every sample. 

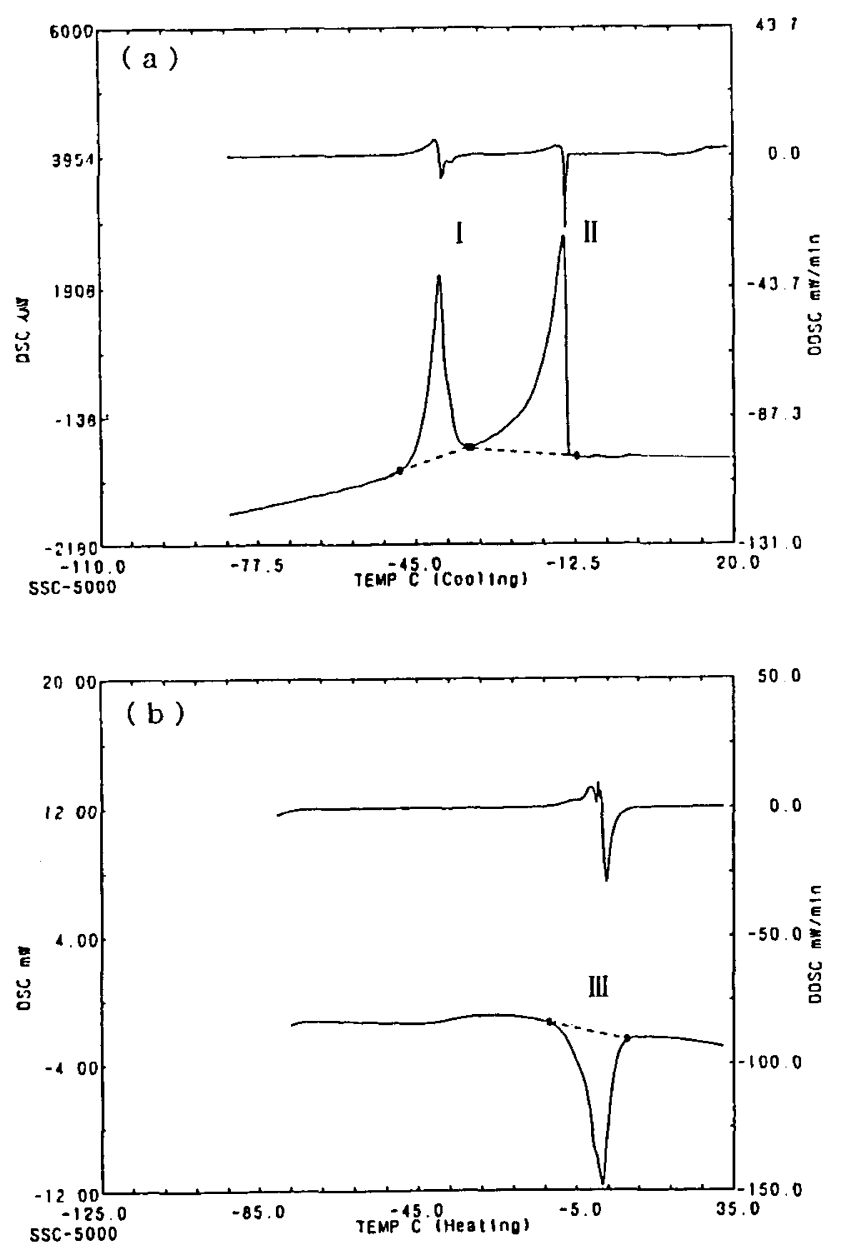

Figure 3. DSC thermograms illustrating the heating and cooling behavior the synthetic copolymer gel (sample's No. 6). The free water and constrained water are shown the peaks at temperatures near $-15^{\circ} \mathrm{C}$ and $-42^{\circ} \mathrm{C}$ in (a). The heat of fusion is shown the peak at temperatures near $0^{\circ} \mathrm{C}$ in $(\mathrm{b})$.

I : constrained water, II : free water, III : heat of fusion.

The STR values were defined as the percentage of signal loss between unsaturated (i.e., SPGR) and saturated (ST-SPGR) images according to the eq 1 ;

$$
\operatorname{STR}(\%)=100 \times\left(1-M_{\mathrm{s}} / M_{0}\right)
$$

where $M_{0}$ and $M_{\mathrm{s}}$ are the signal intensity on the SPGR and on the ST-SPGR image, respectively. The STR-75, -19 , and -4 represent STR values at 75,19 , and $4 \mathrm{ppm}$ apart from off-resonance frequency of the water, respectively. The ROI was ellipsoid and possessed a sampling area of $13 \mathrm{~mm}^{2}$.

To confirm the correlation between STR values and the water contents in the copolymer gels, the reliability ( $p$-value) in Mann-Whitney's U-test were used in this study. The $p$-values less than 0.05 should be requested for the significant data.

\section{RESULTS AND DISCUSSION}

Water structure in synthetic polymer gels without destruction of the samples has been also studied by means of conventional NMR method as described above. ${ }^{1-7}$ Recently the interaction of water with polymer matrixes,

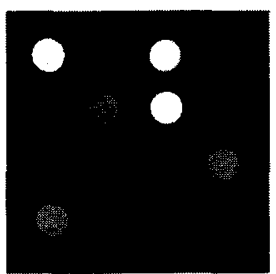

(a)

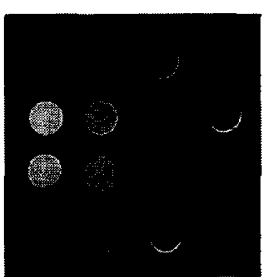

(b)

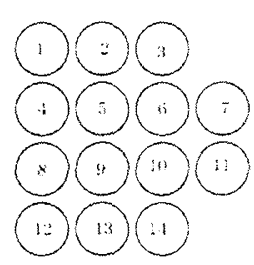

(c)
Figure 4. Conventional MR images of the synthetic copolymer gels. (a) $\mathrm{T}_{1}$-weighted image. (b) $\mathrm{T}_{2}$-weighted image. (c) Sample No. in the image.

mainly in the term of hydrogen bonding, has been investigated through chemical shifts of ${ }^{17} \mathrm{O}$ nucleus resonance. ${ }^{19}$ However, the apparatus for this application is not so popular.

Hence we attempted to assess the saturation transfer ratios (STR) for $\mathrm{T}_{1}$-weighted imaging of the synthetic polymer gels, irradiating three different off-resonance pulses at 75,19 , and $4 \mathrm{ppm}$ apart from the center position of water resonance. The purpose of this study is to examine the correlation between STR values in the MRI measurement and other physical or chemical parameters by which the hydrophilicity of the polymer gels was described and to suggest any principles, if they would be found, for the characterization of the gels.

Figure 3 shows the most typical DSC curve for heating (a) and cooling (b) of the gel of sample number 6 . The free water and constrained water are shown the peaks at near -12 and $-40^{\circ} \mathrm{C}$, respectively, in the cooling process. However only one peak appears around $0^{\circ} \mathrm{C}$ in the heating process, fusing frozen-water. The amounts of freezable water can be estimated from the heat of fusion of the peak in the heating process on the basis of fusing enthalpy of water $\left(333.4 \mathrm{~J} \mathrm{~g}^{-1}\right)$. It is suggested that several gels must contain considerable amounts of nonfreezing water which is not detected in DSC, because the summation of free and constrained water contents are often short of the total amounts of water contents determined by "drying-up method".

The total water contents determined by drying-up method in the samples and free water contents estimated by DSC method are summarized in Table I, in which the solubility parameter (SP) of each sample copolymer is given at the last column, too. SP values are calculated by using Fedors' contribution parameter ${ }^{20}$ based on data of heat of evaporation. Irrespective of a different chemical structure, samples No. 1, 3, 6, and 11 contained nearly equal amount of water in total, but free and bound water contents were different from each other.

The $\mathrm{T}_{1}$ (longitude relaxation time)-weighted and $\mathrm{T}_{2}$ (transverse relaxation time)-weighted images are shown in Figure 4. Also these images are able to supply certain information concerning the samples. However the signal intensity of these images did not show clear correlation with the total water contents (Figure 5), because their signal intensity were obtained from $\mathrm{T}_{1}$ or $\mathrm{T}_{2}$ weighted images do not reflect pure $\mathrm{T}_{1}$ or $\mathrm{T}_{2}$ relaxation time itself.

In addition to (a) conventional SPGR and (b) ST-SPGR at the frequency offset by $4 \mathrm{ppm}$ from water proton, (c) 
Table I. Characterization of sample gels

\begin{tabular}{|c|c|c|c|c|c|c|c|}
\hline \multirow{2}{*}{$\begin{array}{l}\text { Samples } \\
\text { No. }\end{array}$} & \multirow{2}{*}{$\begin{array}{l}\text { Composition } \\
\text { (\% of unit) }\end{array}$} & \multicolumn{5}{|c|}{ Water Content /\% } & \multirow{2}{*}{$\begin{array}{c}\text { Solubility Parameter } \\
\left(\mathrm{MJ} \mathrm{m}^{-3}\right) 1 / 2\end{array}$} \\
\hline & & Total & Free & Bound $^{a}$ & [Constrained, & Non-Frozen ${ }^{a}$ ] & \\
\hline 1 & $\operatorname{HEMA}(65)$, GMA(35) & 58.0 & 8.0 & 50.0 & {$[14.7$,} & $35.3]$ & 24.78 \\
\hline 2 & HEMA(83), GMA(17) & 48.0 & 7.1 & 40.9 & {$[4.2$} & $36.7]$ & 25.89 \\
\hline 3 & $\mathrm{GMA}(32), N-\mathrm{VP}(41), \operatorname{MMA}(27)$ & 59.0 & 2.9 & 56.1 & {$[9.8$} & $46.3]$ & 22.93 \\
\hline 4 & $\operatorname{HEMA}(20), N-\mathrm{VP}(80)$ & 83.0 & 66.2 & 16.8 & {$[0.0$} & $16.8]$ & 26.69 \\
\hline 5 & $\operatorname{HEMA}(40), N-\operatorname{VP}(60)$ & 74.0 & 52.0 & 22.0 & 0.0 & $22.0]$ & 26.76 \\
\hline 6 & $\operatorname{HEMA}(60), N-\operatorname{VP}(40)$ & 61.0 & 7.5 & 53.5 & [ 9.5 & $44.0]$ & 26.81 \\
\hline 7 & $\operatorname{HEMA}(80), N-\mathrm{VP}(20)$ & 47.0 & 6.9 & 40.1 & {$[3.2$} & $36.9]$ & 26.89 \\
\hline 8 & $N-\mathrm{VP}(88), \operatorname{MMA}(4), \mathrm{BzMA}(8)$ & 79.2 & 58.6 & 20.6 & {$[0.0$} & $20.6]$ & 25.93 \\
\hline 9 & $N$-VP(79), MMA(13), BzMA(8) & 73.2 & 51.3 & 21.9 & 0.0 & 21.9] & 25.33 \\
\hline 10 & $N-\operatorname{VP}(70), \operatorname{MMA}(22), \operatorname{BzMA}(8)$ & 64.7 & 42.6 & 22.1 & {$[0.0$} & $22.1]$ & 24.70 \\
\hline 11 & $N-\operatorname{VP}(61), \operatorname{MMA}(34), \operatorname{BzMA}(5)$ & 55.0 & 22.0 & 33.0 & {$[0.0$} & $33.0]$ & 24.06 \\
\hline 12 & $N-\operatorname{VP}(52), \operatorname{MMA}(40), \operatorname{BzMA}(8)$ & 40.0 & 7.6 & 32.4 & {$[0.0$} & $32.4]$ & 23.50 \\
\hline 13 & $N-\operatorname{VP}(43), \operatorname{MMA}(49), \operatorname{BzMA}(8)$ & 28.5 & 0.0 & 28.5 & {$[0.0$} & $28.5]$ & 22.88 \\
\hline 14 & $N-\operatorname{VP}(34), \operatorname{MMA}(58), \operatorname{BzMA}(8)$ & 18.4 & 0.0 & 18.4 & {$[1.1$} & $17.3]$ & 22.27 \\
\hline
\end{tabular}
${ }^{\mathrm{a}}$ See Text.

(a)

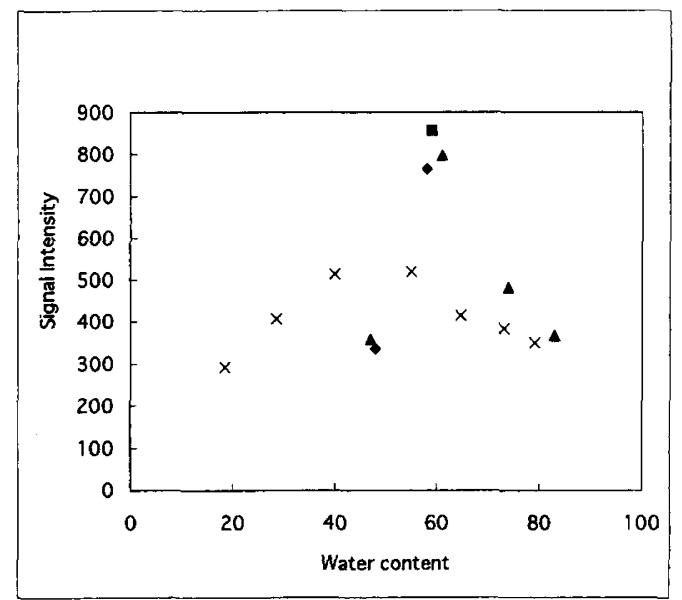

(b)

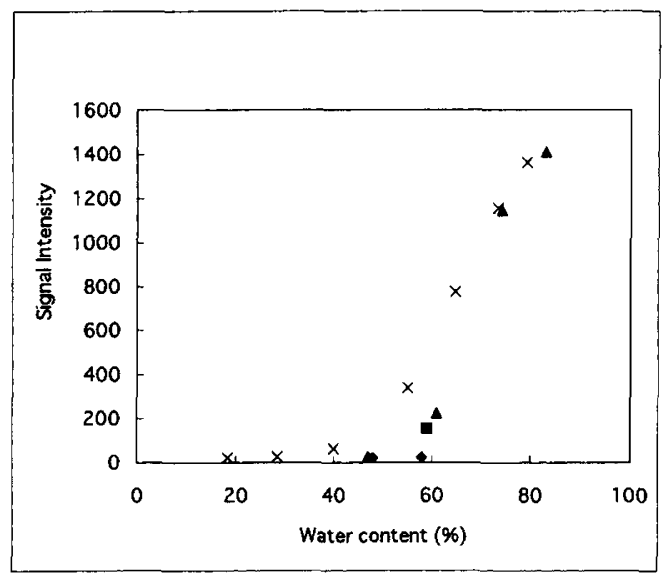

Figure 5. Correlation between signal intensity of $\mathrm{T}_{1}$-weighted, $\mathrm{T}_{2}$-weighted images and total water content in the synthetic copolymer gels. (a) Correlation between signal intensity of $\mathrm{T}_{1}$-weighted image and total water content. (b) Correlation between signal intensity of $\mathrm{T}_{2}$-weighted image and total water content.

$\checkmark$ : poly (HEMA-stat-GMA)s, $\square$ : poly (N-VP-stat-GMA / MMA)s.

$\Delta$ : poly (HEMA-stat-N-VP)s, $\times$ : poly (N-VP-stat-MMA / BzMA)s.

the calculated STR-4 images for the samples are shown in Figure 6. The conventional SPGR imaging of the samples were directly resulted from $\mathrm{T}_{1}$-weighted image. In the ST-SPGR images, the signal intensity was lower (a)
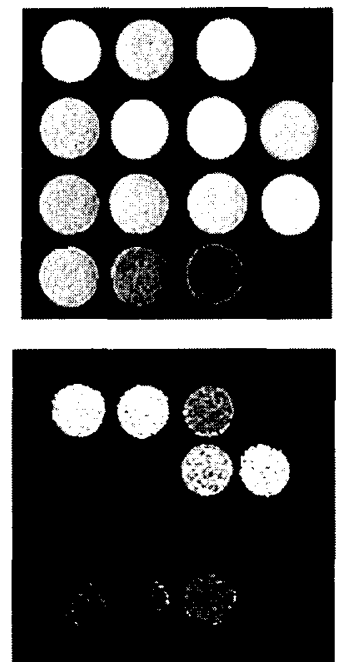

(d)

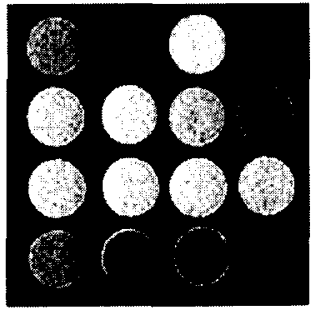

(b)

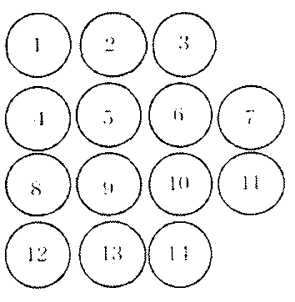

Figure 6. MR images of the synthetic copolymer gels with saturation transfer effect. (a) Conventional SPGR image (resulting $\mathrm{T}_{1^{-}}$ weighted image). (b) ST-SPGR image at frequency offset of 4-ppm. (c) Calculated STR image at frequency offset of 4-ppm. The STR image was calculated from conventional SPGR image (a) and STSPGR image (b) with the eq 1. (d) Sample No in the image.

than that in the conventional SPGR. Such reduction of the signal intensity could be just expected as "ST-effect". The ST-effect is larger, the intensity in the STR image become higher, as is demonstrated by the STR-4 images calculated according to the eq 1.

It is worth pointing that the degree of reduction should be dependence upon the frequency of ST pulse. The contrast of the imaging observed in this study must be important in evaluating the hydrophilicity in the synthetic copolymer gels. The STR values for the samples are correlated with their total water content as Figure 7 [(a) STR-75, (b) STR-19, and (c) STR-4]. The STR-75 values show a correlation curve regardless of the samples and increased with decreasing total water contents in the synthetic gels. Now we would propose that STR-75 values and its imaging can be applied to the estimation for total contents of water irrespective of the water conditions in a gel network. In the case of STR-19, however, 
Table II. STRs of the synthetic copolymer gels

\begin{tabular}{|c|c|c|c|c|c|c|c|}
\hline \multirow{2}{*}{ Samples } & \multirow{2}{*}{$\mathrm{n}$} & STR-75 & \multirow{2}{*}{ MW-test } & STR-19 & \multirow{2}{*}{ MW-test } & STR-4 & \multirow{2}{*}{ MW-test } \\
\hline & & Average $\pm \mathrm{SD} / \%$ & & Average $\pm \mathrm{SD} / \%$ & & Average $\pm \mathrm{SD} / \%$ & \\
\hline Hydrophilic gels & 7 & $6.55 \pm 4.96$ & \multirow{2}{*}{ No Significant } & $20.06 \pm 11.21$ & \multirow{2}{*}{ No Significant } & $42.89 \pm 19.12$ & \multirow[b]{2}{*}{$p<0.05$} \\
\hline Hydrophobic gels & 7 & $7.89 \pm 4.91$ & & $9.69 \pm 5.98$ & & $23.53 \pm 13.27$ & \\
\hline
\end{tabular}

Values are means \pm standard deviation. P-values were determined using the Mann-Whitney U-test.

(a)

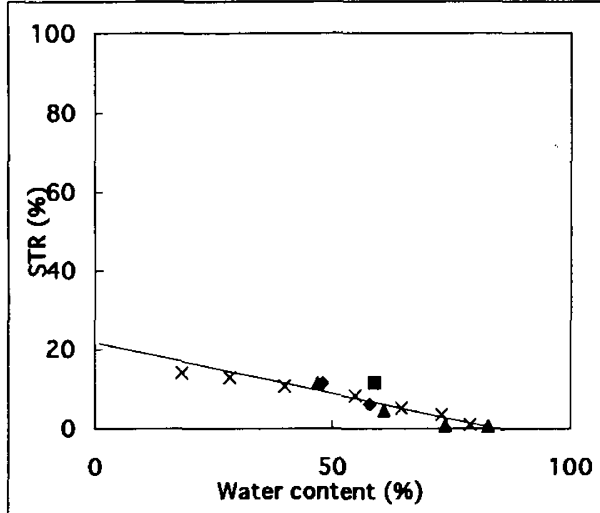

(b)

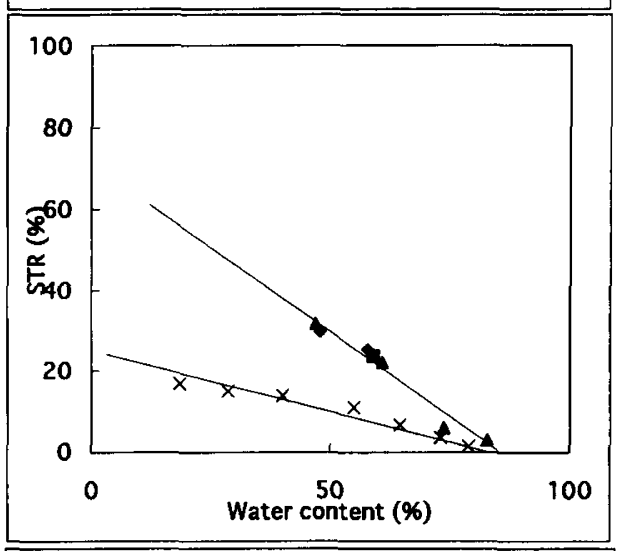

(c)

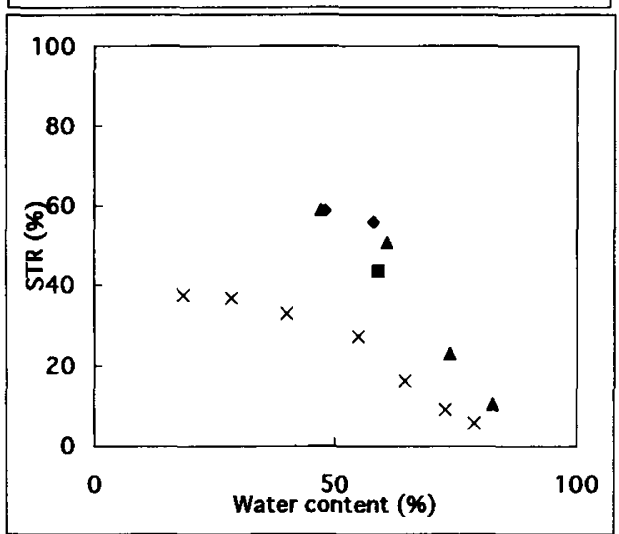

Figure 7. Correlation between STRs and total water content in the synthetic copolymer gels. (a) Correlation between STR and total water content at frequency offset of 75-ppm. (b) Correlation between STR and total water content at frequency offset of 19-ppm. (c) Correlation between STR and total water content at frequency offset of 4-ppm.

$\checkmark$ : poly (HEMA-stat-GMA)s, $\square$ : poly (N-VP-stat-GMA / MMA)s

A: poly (HEMA-stat-N-VP)s, $X$ : poly (N-VP-stat-MMA/BzMA)s.

two linear relationships were found out, dividing the samples into the gels symbolized as $\boldsymbol{\nabla}, \boldsymbol{\nabla}$, and $\boldsymbol{\Delta}$ and remaining ones symbolized as $X$. Also the STR-4 values could be separated to two groups, but one group did not show the linear correlation.

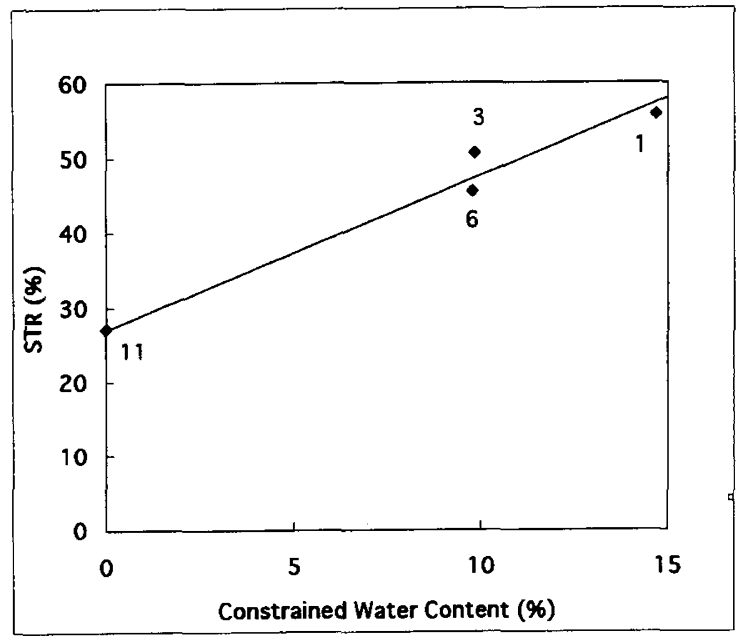

Figure 8. Correlation between STR and constrained water content in the synthetic copolymer gels with the nearly equal total water content (ca. $60 \%$ ) at frequency offset of 4-ppm (sample No. 1, 3,6 , and 11).

According to the averaged STR-19 and -4 values summarized in Table II, the essentially hydrophilic copolymers ( $\boldsymbol{\nabla}, \boldsymbol{\square}$, and $\boldsymbol{\Delta}$ ) can be always characterized by the considerably higher STR value than that for hydrophobic one $(X)$ even though they involved the same amounts of water as a whole. STR-19 values showed good correlation with the total water contents in the samples, moreover classified the sample gels into two groups, hydrophilic or hydrophobic polymer matrixes, depending on the numerical level of STR. Therefore we believe that STR-19 values and its imaging can be useful to discuss the fundamental states of the gel with water. The STR-4 values are found to be more separable parameter depending on the different hydrophilicity of the samples. It seems that each synthetic gels showed individually specific STR-4 values in dependence with their chemical structure, too.

However, further research is necessary to elucidate the potential relationship between STR-4 values and chemical structure of the copolymers. Era et al. have already pointed out that the ST-effect at the offsetfrequency below $10 \mathrm{ppm}$ apart from the water resonance frequency (usually $4.70 \mathrm{ppm}$ from TSP) may correlate with the number of hydroxyl group. ${ }^{21,22}$ They also suggested that the ST-effect at the offset-frequency more than $20 \mathrm{ppm}$ from the water resonance might reflect the molecular size-weighted magnetization transfer. ${ }^{21,22}$

In order to find other new facts, the data for the synthetic gels having nearly equal total water contents but chemically different structure are compared in Figure 8. At first, there is a good correlation between STR-4 values and "constrained" water contents in the sample ( $r=$ 0.93). Next, the relationship between STR-4 values of 


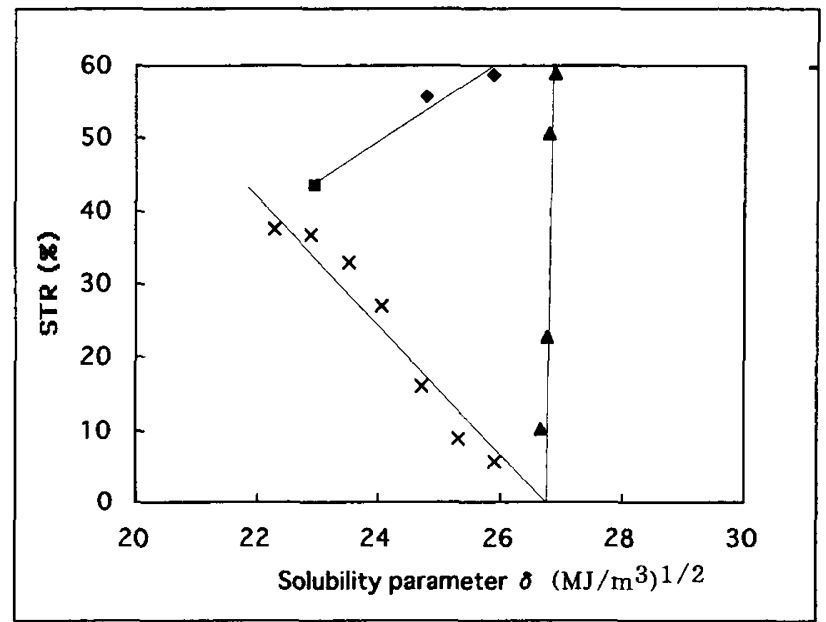

Figure 9. Correlation between STR and solubility parameter in the synthetic copolymer gels at frequency offset of 4-ppm.

$\checkmark$ : poly (HEMA-stat-GMA)s, $\mathbf{\square}$ : poly (N-VP-stat-GMA / MMA)s

$\Delta$ : poly (HEMA-stat-N-VP)s, $\times$ : poly (N-VP-stat-MMA / BzMA)s.

various samples and their solubility parameters was investigated. Fedors' SP values means in brief the summation of the partial contribution of each structural component for enthalpy in the course of releasing water from matrixes, in other words, for the degree of constraining force of the copolymer for water. As shown in Figure 9, remarkably good correlation can be observed for each copolymer series. For example, the correlation coefficients $(r)$ were 0.92 and 0.95 for poly(HEMA-stat-N-VP)s $(\boldsymbol{\Delta})$ and poly(N-VP-stat-MMA/BzMA)s $(X)$, respectively. In the latter which are recognized as the typical hydrophobic gel, STR-4 values are in inverse proportion to the solubility parameter of each sample. On the contrary, STR-4 values for the hydrophilic gels are directly proportional to their solubility parameter. It should be worthy of note that the slope of the correlation line for a series of poly(HEMA-stat-N-VP)s is steeper than that of poly(HEMA-stat-GMA)s and poly(N-VP-stat-GMA/MMA)s.

Thus we insist on the above results that the STR-4 value and STR-4 image must be a reliable numerical index and visible information about the condition of water in the synthetic gels, respectively. Accumulation of STR data for various types of the sample in future would bring to us the variable information concerning total water contents, free water contents, and bound water contents in the synthetic polymer gels. Further the connection of STR value with the solubility parameter would also support to account for relationship between their chemical structure and hydrophilicity of the hydrogels.

\section{CONCLUSIONS}

Through MRI measurement for various synthetic copolymer gels, it was found that STR-75, -19, and -4 values were able to adopt as a new parameter in evaluating hydrophilic character of the samples. The higher value of STR-19 or -4 is associated with higher hydrophilicity of the sample. The values of STR-19 were correlating with the total water contents, moreover separable the samples into two groups, hydrophilic or hydrophobic gel as a whole. STR-4 values are correlated with not only the total water content but also its conditions in more detail. The close relationship between STR-4 values with the solubility parameter was found for the first time, meaning that these values were reflected by chemical structure of the gels, too. Therefore these STR values and STR images in MRI measurement would be a new useful tool for evaluation of the hydrophilicity of the synthetic copolymer gels without invasive process to the samples. The MRI will be recognized as a good method not only medical field but also industrial field for the analysis.

Acknowledgment. The first author thanks Dr. Nobukazu Fuwa and Dr. Yukio Uchiyama at Aichi Cancer Center for their valuable advice.

\section{REFERENCES}

1. N. Murase and T. Watanabe, Magn. Reson. Med., 9, 1 (1989).

2. Y. P. Zhang, R. N. Lewis, and R. N. McElhaney, Biophys. J., 72, 79 (1997).

3. L. J. Peschier, J. A. Bouwstra, J. de Bleyser, H. E. Junginger, and J. C. Leyte, Biomaterials, 14, 945 (1993).

4. P. S. Belton. Int. J. Biol. Macromol., 21, 81 (1997).

5. M. C. Vackier, B. P. Hills, and D. N. Rutledge, J. Magn. Reson., 138, 36 (1999).

6. T. Watanabe, N. Murase, M. Staemmler, and K. Gersonde, Magn. Reson. Med., 27, 118 (1992).

7. H. Yang and T. Schleich, J. Magn. Reson. B, 105, 205 (1994).

8. M. Sogami, S. Nagaoka, S. Era, H. Inoue, K. Kuwata, K. Kida, H. Watari, K. Shiga, and K. Akasaka, Int. J. Peptide Protein Res., 28, 130 (1986).

9. K. Akasaka, R. Ishima, and S. Shibata, Physica B, 164, 163 (1990).

10. P. R. Moran and C. A. Hamilton, Magn. Reson. Imaging, 13, 837 (1995).

11. J. L. Ulmer, V. P. Mathews, C. A. Hamilton, A. D. Elster, and P. R. Moran, AJNR, 17, 805 (1996).

12. R. S. Balaban, First Nottingham Symposium on Magnetic Resonance in Medicine, Prog., P 07, 4 (1994).

13. R. E. Sepponen, "Magnetic resonance imaging," 2nd ed, Mosby-Year Book, St. Louis, MO, 1992, pp 204-218.

14. F. W. Flickinger, J. D. Allison, R. M. Sherry, and J. C. Wright., Magn Reson Imaging, 11, 617 (1993).

15. Orel S. Greenstein, M. D. Schnall, V. A. LiVolsi, and R. H. Troupin, Radiology, 190, 485 (1994).

16. E. S. Fobben, C. Z. Rubin, L. Kalisher, A. G. Dembner, M. H. Seltzer, and E. J. Santoro, Radiology, 196, 143 (1995).

17. P. C. Stomper, S. Herman, D. L. Klippenstein, J. S. Winston, S. B. Edge, and M. A. Arredondo, Radiology, 197, 387 (1995).

18. W. E. Roorda, J. A. Bouwstra, M. A. de Vries, and H. E. Junginer, Biomaterials, 9, 494 (1988).

19. Y. Machida, S. Kuroki, M. Kanekiyo, M. Kobayashi, I. Ando, and S. Amiya, Reports on Progress in Polymer Physics in Japan, 42, 75 (1999).

20. R. F. Fedors, Polym. Eng. Sci., 14, 147 (1974).

21. S. Era, M. Sogami, Y. Kinosada, S. Matsushima, K. Kato, and N. Nagai, Proc. Int. Workshop Appli. Magn. Reson. Other Spectrosc. Tech. Food Sci. (MRF) Abstract, 107 (1997).

22. M. Sogami, S. Era, T. Koseki, and N. Nagai, J. Peptide Res., 50, 465 (1997). 